\title{
Die Stimmung kippt (nicht von allein)
}

\section{Sprachliche Angstkonstruktion im Flüchtlingsdiskurs}

\begin{abstract}
Der Beitrag beschäftigt sich aus diskurslinguistischer Sicht mit den sprachlichen Mechanismen der Angstkonstruktion im aktuellen Flüchtlingsdiskurs. Ausgehend von der Annahme, dass Angst ein massenmedial konstruiertes Phänomen darstellt, werden formelhafte Wendungen in ihrer angstkonstruierenden Funktion innerhalb von Argumentationsmustern (Topoi) untersucht. Die Analyse zeigt, dass den formelhaften Wendungen zum einen eine angstschürende, zum anderen aber eine kritisch-distanzierende Funktion zukommt, während sie innerhalb von Topoi entweder als zentrale Realisierungsmittel fungieren oder die sprachliche Realisierung von Argumentationsmustern stützen.

Using the discourse-linguistic approach, this paper examines the construction of fear in the current discourse on refugees in Germany. Based on the assumption that fear is constructed by mass media, formulaic patterns are analyzed with regard to their role in creating fear of refugees within argumentation patterns (topoi). The findings indicate that the formulaic patterns fuel fear and mistrust on the one hand, but on the other hand, they are also used to criticize the construction of fear of refugees. Within topoi they serve as either central or marginal linguistic means of its realization.
\end{abstract}

\section{Einstieg}

Der vorliegende Beitrag greift das Thema Angst aus sprachwissenschaftlicher Perspektive auf und widmet sich den sprachlichen Konstruktionsmechanismen dieses Phänomens auf der Diskursebene. Ausgehend von dem Verständnis von Diskurs als einer thematisch und zeitlich abgegrenzten Textmenge (vgl. Busse \& Teubert 1994) wird Angst im Rahmen des jüngsten Migrationsdiskurses zwischen 2015 und 2017 analysiert. Als Ausgangspunkt der Untersuchung dient die These von Bauman (2016: 7), dass die Berichterstattung über die aktuelle Migration durch eine moralische Panik gekennzeichnet ist, die sich unter anderem in der Konstruktion der Angst vor dem Fremden niederschlägt. Somit stehen Migrationsängste als eine spezifische Angstform im Vordergrund, die im Hinblick auf implizite sprachliche Mittel der Angstkonstruktion betrachtet werden. Was 
darunter verstanden wird, lässt sich an folgenden Textausschnitten aus den Massenmedien nachvollziehen:

(1) Ganz oft hört man: Die Flüchtlinge bedrohen den Wohlstand unserer Gemeinde. Oder: Dann finde ich keinen Kita-Platz mehr für mein Kind. Die Menschen fühlen eine große Unsicherheit und versuchen, Erklärungen zu finden. Häufig bleibt unausgesprochen, dass dahinter andere Formen der Bedrohung stehen. In der Psychologie sprechen wir von symbolischer Bedrohung. Die Leute haben den Eindruck: Jetzt geht die deutsche Kultur den Bach runter. (SPON 15.09.2015, „Man muss die Angst ernst nehmen“)

(2) (...) Die größte Herausforderung seit der deutschen Einheit. Wir sind in einer Notlage. Europa stürzt ins Chaos. Unsere Gesellschaft wird sich verändern. Wie aber wird es dann sein: ungemütlicher, enger? Aufregender ganz sicher. Doch wer mag sich heute schon aufregen? (FAZ 19.09.2015, „Das Ende der kleinen, heilen Welt“)

Im ersten Beispiel stehen verschiedene Bedrohungsszenarien im Vordergrund. Der Aufnahmegesellschaft werden Sozialneid (vgl. Die Flüchtlinge bedrohen den Wohlstand unserer Gemeinde. Dann finde ich keinen Kita-Platz für mein Kind) und Ängste vor kulturellen Veränderungen (vgl. Jetzt geht die deutsche Kultur den Bach runter) angesichts der Flüchtlingsaufnahme zugeschrieben. Außerdem ist die Angstkonstruktion an den Lexemen Bedrohung und Unsicherheit erkennbar. Im zweiten Beispiel lässt sich Angst am Singularitätstopos ${ }^{1}$ (die größte Herausforderung seit der deutschen Einheit) erkennen. Darüber hinaus entsteht durch kurze parataktische, dicht aufeinander folgende Sätze der Eindruck der Unvermeidbarkeit der befürchteten Veränderungen. Schließlich kommt Ungewissheit durch zwei rhetorische Fragen zum Tragen. Auf diese Weise sieht man, dass sich die Veränderung auf die fehlende Vertrautheit mit der neu entstandenen Situation bezieht.

In den beiden zitierten Belegen kommt das Lexem Angst nicht vor, dennoch ist die Angstkonstruktion an anderen sprachlichen Mitteln erkennbar. Solche sprachlichen Mechanismen kommen im massenmedialen Flüchtlingsdiskurs immer wieder vor und lassen sich unterschiedlichen Ebenen der Musterhaf-

1 Vgl. dazu Römer \& Wengeler (2013: 272-273). 
tigkeit von Phraseologismen über Wortbildungsmuster bis hin zu den Argumentationsmustern und Metaphern (vgl. dazu Stein \& Stumpf 2019: 18) zuordnen. ${ }^{2}$

Die implizite Angstkonstruktion im aktuellen Migrationsdiskurs stellt den Untersuchungsgegenstand des vorliegenden Beitrags dar. Genauer gesagt werden formelhafte Wendungen (vgl. Filatkina 2018a, s. dazu weiter unten) als sprachliche Realisierungsmittel von Argumentationsmustern oder Topoi (vgl. Wengeler 2003, s. unten) in den Blick genommen. Ihre Analyse soll deutlich machen, wie Migrationsängste implizit konstruiert werden sowie welche diskursive Funktion den formelhaften Wendungen bei der Angstkonstruktion zukommt.

Die Analyse stellt einen Beitrag zur kulturwissenschaftlichen Angstforschung dar und zeigt die Möglichkeiten ihrer Erweiterung um diskurslinguistische Perspektiven auf. Im Sinne der Diskurssemantik (vgl. Busse 1987) geht es darum, durch die Aufdeckung von sprachlichen Mechanismen der Angstkonstruktion im Flüchtlingsdiskurs die kollektiven angstbesetzten Wissensbestände mit diskurslinguistischen Methoden zu erschließen, um auf diese Weise etwas über die Mentalitäten bzw. über das kollektive Denken, Fühlen und Wollen (vgl. Hermanns 1995: 77) einer Gesellschaft zu erfahren.

Der Beitrag gliedert sich wie folgt: Zunächst wird der Forschungsstand zur diskurslinguistischen Angstforschung skizziert, wobei der Fokus auf den Migrationsdiskursen liegt. Anschließend werden das Analysemodell und das Untersuchungskorpus vorgestellt. Die Korpusanalyse widmet sich der Frage nach den diskursiven Funktionen von formelhaften Wendungen als sprachlichen Realisierungsformen von Argumentationsmustern in einem Korpus von Zeitungstexten zur aktuellen Flüchtlingsdebatte. Im Fazit werden drei unterschiedliche Aspekte der diskursiven Funktionen, die im untersuchten Korpus durch formelhafte Wendungen ermittelt wurden zusammenfassend aufgegriffen.

2 Die sprachlichen Mechanismen der Angstkonstruktion lassen sich auch durch die Unterscheidung in punktuelle und flächige Bedeutungsbildung (vgl. Gardt 2013: 19-20) erklären. So kommt die punktuelle Angstkonstruktion durch einzelne lexikalische Mittel zustande (wie etwa Panik oder Notlage), während die flächige Angstkonstruktion u. a. durch Topoi erfolgt. 


\section{Angst als diskurslinguistischer Unter- suchungsgegenstand}

Im Unterschied zur psychologischen Angstforschung, die sich hauptsächlich auf individuelle Angst als physisches Phänomen konzentriert (vgl. u.a. Bandelow 2004), wird in der kulturwissenschaftlichen Angstforschung davon ausgegangen, dass Ängste „über Jahrtausende hinweg geformt, geprägt, gelenkt, stilisiert - unterdrückt und diszipliniert oder gepflegt und kultiviert“ (Bergmann 2002: 1-2) werden. Somit wird Angst einerseits als körperliches andererseits aber auch als kulturelles Phänomen verstanden, das unter anderem durch die Massenmedien geprägt ist. Die massenmediale Perspektive steht im Vordergrund dieses Beitrags.

Dabei wird die konstruktivistische These vertreten, dass Migrationsängste sprachlich konstruierte Phänomene sind. Das bedeutet aber nicht, dass die Existenz von migrationsbezogenen Ängsten negiert wird (vgl. dazu Gardt 2018: 21). Dennoch ist mit Furedi (2007: 3) davon auszugehen, dass ,individual fears are cultivated through the media and are less and less the outcome of direct experience. Fear is decreasingly experienced first-hand and increasingly experienced on a discursive and abstract level [...]“. Auch Altheide (2002: 6) stellt fest, ,that the mass media and popular culture are the most important contributors to fear".

In einem engeren Sinne ist die vorliegende Analyse der diskurslinguistischen ${ }^{3}$ Beschäftigung mit Angst zuzuordnen. Obwohl Angst auf nahezu allen sprachlichen Ebenen untersucht wurde ${ }^{4}$ und trotz der Tatsache, dass sie längst als kulturwissenschaftliche Disziplin gilt (vgl. Wengeler 2006), wird die Sprachwissenschaft aus der interdisziplinären kulturwissenschaftlichen Angst-

3 Gemeint ist der Diskursbegriff im Sinne der Diskurssemantik (vgl. dazu Busse 1987, Wengeler 2005) bzw. seine praktische Umsetzung in der Begriffsbestimmung von Busse \& Teubert (1994).

4 Vgl. u. a. zur sprachwissenschaftlichen Untersuchung des Angstwortschatzes Bergenholz (1980) sowie zum Angstwortschatz aus diachroner Perspektive Filatkina (2016). 
forschung weitgehend ausgeklammert ${ }^{5}$. Mit diesem Beitrag soll gezeigt werden, dass auch die diskurslinguistische Perspektive im Rahmen der kulturwissenschaftlichen Angstforschung zu verorten sind.

In mehreren Untersuchungen wurden bereits mehrere methodische Vorschläge zur diskurslinguistischen Analyse des Phänomens Angst herausgearbeitet. Wohl als erste umfangreiche diskurslinguistische Untersuchung (zumindest im deutschsprachigen Raum) gilt die Dissertation von Radeiski (2011). Am Beispiel der massenmedialen Berichterstattung zur Vogelgrippe im Jahr 2006 werden als zentrale Analysekategorien drei diskursive Rollen eingeführt, und zwar Angsterzeugung (Angstkonstruktion durch massenmediale Vermittlung, die diskursive Existenz der Angst vor Virus), Angstzuschreibung (ein Subjekt, das sich Angst einjagen lässt) und Angstbewältigung (die Begrenzung des Grades der diskursiven Angst) (vgl. Radeiski 2011: 157-183). Wie noch gezeigt wird, lassen sich diese drei Aspekte teilweise auch auf die vorliegende Untersuchung übertragen. Filatkina (2015) legt ein methodisches Instrumentarium zur diskurshistorischen Analyse des Begriffs Zukunftsangst vor. Das Analyseraster besteht aus zehn in Anlehnung an den begrifflichen Apparat von Peter von Polenz und die Theorie der semantischen Rollen benannteKategorien (vgl. Filatkina 2015: 81-82). Es wurde an einem umfangreichen Korpus von Zeitungsartikeln aus Spiegel-Online zwischen 1970 und 2013, in denen das Lexem Zukunftsangst vorkommt, praktisch umgesetzt. Konzentriert sich Filatkina (2015) auf die Analyse eines einzelnen Lexems, wird in Filatkina (2017) ein Analysemodell herausgearbeitet, mit dem die sprachlichen Mechanismen der Angstkonstruktion auf lexikalisch-semantischer, morpho-syntaktischer (sogenannte Grammatik der Angst) sowie auf textueller und diskursiver Ebene sowohl ausgehend vom Lexem Angst als auch ausgehend von einem bestimmten Diskurs, nämlich vom Migrationsdiskurs untersucht wird. Wengeler (2019) stellt diskurslinguistische Methoden für die Untersuchung der sprachlichen Konstruktion von Zukunftsangst und Vertrauen vor und nimmt insbesondere die Krisen- und die Migrationsdiskurse in den Blick. Der Fokus liegt sowohl auf der expliziten als auch auf

5 Das ist unter anderem an den Beiträgen in der bisher umfangreichsten Publikation zur kulturwissenschaftlichen Angstforschung (vgl. Koch 2013) ersichtlich. Dort wird der sprachliche Charakter der Angst zwar betont, aber der Sammelband kommt ganz ohne sprachwissenschaftliche Beiträge aus. Das gilt auch für das DFG-Netzwerk „Spielformen der Angst“, aus dem der erwähnte Sammelband maßgeblich hervorgegangen ist (vgl. dazu Koch 2010). Auch das an der Freien Universität Berlin zwischen 2007 und 2014 angesiedelte Exzellenzcluster „Languages of Emotion“ (vgl. http://www.loe.fu-berlin.de/), das über 70 Forschungsprojekte zur Emotionsforschung umfasst, enthält kein einziges sprachwissenschaftliches Projekt, obwohl es paradoxerweise schon durch den Titel auf die Rolle der Sprache in der Emotionsforschung verweist. 
der impliziten Angst- und Vertrauenskonstruktion. Georgi (2018) ermittelt im Rahmen einer korpuspragmatischen Studie die Bedeutungen des Lexems Angst und untersucht an einem Korpus von Artikeln der Zeit-Online zwischen 2015 und 2017 typische Wortverbindungen und syntaktische Verknüpfungen mit dem Lexem Angst (vgl. Georgi 2018: 120-121) ${ }^{6}$.

Innerhalb der diskurslinguistischen Analysen von Migrationsdiskursen ist die korpuslinguistische Analyse des Begriffs Angst in Massenmedien, Foren und Talkshows von Becker (2015) erwähnenswert, in der der Frage nach der sprachlichen Konstruktion von Migrationsängsten zwischen Juli 2013 und Juni 2014 nachgegangen wird. Die an die Ansätze der Kritischen Diskursanalyse angelehnte Untersuchung der Polit-Talkshows zum Thema Flucht von Goebel (2017: 268-283) widmet sich teilweise den Mechanismen der impliziten Angstkonstruktion. Ähnlich wie bei Becker (2015) wird nicht die Hochphase der aktuellen Flüchtlingsdebatte untersucht, sondern der Zeitraum zwischen 2011 und 2014.

Dass Angst im jüngsten Flüchtlingsdiskurs durch verschiedene sprachliche Mechanismen konstruiert wird, zeigt sich etwa an der Metaphernanalyse von Spieß (2016), in der u. a. der von Wolfgang Schäuble verwendeten LawinenMetapher eine stark negative Bewertung von Migration und eine angstschürende Rolle zugewiesen wird (vgl. Spieß 2016: 70). Kreußler \& Wengeler (2018) verbinden mehrere Analyseebenen (Schlüsselwörter, Metaphern und Topoi) und zeigen, dass diese sprachlichen Mittel in den von ihnen untersuchten Medien auch in sprachkritischen Zusammenhängen verwendet werden. Auf der Ebene der Mehrworteinheiten widmen sich einige empirische Untersuchungen den formelhaften Wendungen (s. dazu unten), die mehr oder weniger implizit auf migrationsbezogene Ängste verweisen. Dabei liegt der Fokus vorwiegend auf Wir schaffen das ${ }^{7}$. Wodak (2016) beschäftigt sich mit der Konstruktion einer „Politik der Angst“ in rechtspopulistischen Kontexten während Gür-Şeker (2018) die Angstkonstruktion im Zusammenhang mit der Pegida-Bewegung untersucht. Die in diskursgeschichtlichen Arbeiten der Düsseldorfer Forscher-

\footnotetext{
6 Vgl. auch Kuck (2018b) zur Konstruktion von Angst in der German-Mut-Kampagne der FDP bei den Bundestagswahlen 2017. Darüber hinaus sind auch mehrere diskurslinguistische Untersuchungen erwähnenswert, in denen Angst keinen zentralen Untersuchungsgegenstand darstellt, die aber solche Themen behandeln, die mehr oder weniger mit Angst zusammenhängen wie etwa die Untersuchungen von Krisendiskursen (vgl. Römer 2017, Kuck 2018a sowie die Beiträge in Wengeler \& Ziem 2013), des Islamdiskurses (vgl. Kalwa 2013) oder der Sicherheitsdiskurse (vgl. Schmidt 2018).

7 Vgl. dazu Rada (2018) und Fábián (2019) sowie zur multimodalen Konstruktion von Wir schaffen das in Karikaturen Stumpf \& Kreuz (2018). In Spieß (2018: 49) wurde Deutschland muss Deutschland bleiben erwähnt.
} 
gruppe (vgl. u. a. Böke 1997, Wengeler 2003, Jung, Wengeler \& Böke 1997) untersuchten sprachlichen Mittel wie etwa der Gefahrentopos, bestimmte Bezeichnungen für Migrant ${ }^{\star} i n n e n$ oder Metaphern lassen sich als implizite sprachliche Indikatoren der Angstkonstruktion in früheren Migrationsdiskursen interpretieren.

\section{Methodisches Vorgehen und Datengrundlage}

Das der empirischen Analyse zugrunde liegende Analysemodell beruht auf den Erkenntnissen der Diskurssemantik (vgl. Busse 1987) und der (soziologischen) Vertrauensforschung und geht von der Annahme aus, dass die sprachliche Konstruktion von Migrationsängsten eng mit der Konstruktion von Misstrauen gegenüber den Flüchtlingen verknüpft ist.

Außerdem ist davon auszugehen, dass es sich bei Misstrauen (genauso wie bei Vertrauen) um ein komplexes Konstrukt handelt, das nicht direkt erfragbar ist, sondern nur durch Indikatoren erschlossen werden kann. Die Indikatoren der sprachlichen Konstruktion des (fehlenden) Vertrauens gegenüber Flüchtlingen wurden aus der Vertrauensforschung (vgl. u.a. Seifert \& Brinkmann 2000) übernommen und inhaltlich an den Untersuchungsgegenstand angepasst. Es handelt sich um folgende Indikatoren, die in der folgenden Tabelle dargestellt sind: ${ }^{8}$

Tab. 1: Vertrauensindikatoren im Flüchtlingsdiskurs

\begin{tabular}{ll}
\hline Erfahrungswissen über Flüchtlinge & $\begin{array}{l}\text { Zukunftserwartungen bezüglich } \\
\text { der Folgen der Flüchtlingsaufnahme }\end{array}$ \\
Vertrauenswürdigkeit der Flüchtlinge & $\begin{array}{l}\text { Erwartungshaltungen an } \\
\text { das Verhalten von Flüchtlingen }\end{array}$ \\
\hline
\end{tabular}

Im Verlauf der Korpuslektüre wurden die Textstellen beliebiger Länge mit diesen Indikatoren im Programm zur Unterstützung der qualitativen Datenanalyse Maxqda annotiert. Außerdem wurden diese Indikatoren inhaltlich ausdifferenziert und um die Kategorie der Vertrauensadressat*innen und Diskursakteur*-

8 Aus Platzgründen kann auf die Beschreibung von Vertrauensindikatoren sowie auf ihre diskurslinguistische Operationalisierung nicht eingegangen werden (vgl. dazu ausführlich Belosevic i. V.). 
innen ${ }^{9}$ erweitert. In einem zweiten Schritt wurden in den mit den Vertrauensindikatoren annotierten Textstellen Topoi ermittelt und ebenso in Maxqda annotiert. ${ }^{10}$ Topoi oder Argumentationsmuster werden als „argumentative Muster, die bei der öffentlichen Konstruktion des „Wissens“ gehäuft, seriell vorkommen [...], die nicht immer in gleicher Weise sprachlich materialisiert werden müssen, die aber in vielen Texten als immer wieder ähnlich vorkommende, auf Plausibilität zielende Herstellung von Sachverhaltszusammenhängen erscheinen“ (Römer \& Wengeler 2013: 270) verstanden.

Die Indikatoren des (fehlenden) Vertrauens gegenüber Flüchtlingen können durch unterschiedliche diskurslinguistische Analyseeinheiten „gefüllt“ werden wie etwa durch Schlüsselwörter, Metaphern, Phraseologismen oder Topoi. Die Topoi stellen eine sehr abstrakte Ebene der musterhaft vorkommenden sprachlichen Mittel dar, mit denen migrationsbezogenes Misstrauen und Ängste implizit vor dem Hintergrund der Argumentation pro oder contra Flüchtlingsaufnahme konstruiert werden. Da sie auf sprachlicher Oberfläche unterschiedlich realisiert werden, ist darüber hinaus davon auszugehen, „dass bereits die Verwendung bestimmter zentraler Schlüsselwörter oder Leitvokabeln bzw. einer bestimmten Metaphorik oder eines Phraseologismus das Vorhandensein eines Topos nahe legt“ (Wengeler 2003: 198, Herv. i. O.). Für die hier verfolgte Fragestellung eignen sich alle im Zitat genannten diskurslinguistischen Analyseeinheiten. Während aber Schlüsselwörter und Metaphern als sprachliche Realisierungsformen der Argumentationsmuster mehrfach untersucht wurden, wurden formelhafte Wendungen bisher nur teilweise als diskurslinguistische Analyseeinheiten berücksichtigt ${ }^{11}$. Deswegen steht ihr sprachkonstruierendes Potenzial im Rahmen der vorliegenden empirischen Analyse im Vordergrund, obwohl die Angstkonstruktion im untersuchten Korpus genauso durch die Metaphern

9 Unter dem Indikator Diskursakteur*innen ist die Frage Wer spricht? gemeint bzw. es handelt sich um „die Handelnden im Diskurs, die Wissen hervorbringen, zum Verschwinden bringen, regulieren und befördern“ (Spitzmüller \& Warnke 2011: 137). Als Vertrauensadressat^innen werden die sozialen Gruppen verstanden, die in die Rolle der Vertrauensgeber`innen oder Vertrauensnehmer*innen versetzt werden.

10 Topoi bieten sich für die diskurslinguistische Operationalisierung der impliziten Vertrauens- und Misstrauenskonstruktion im Flüchtlingsdiskurs insofern an, weil die Toposanalyse „Zugriff auf Nichtgesagtes, nur Mitgemeintes, aber dennoch Kommuniziertes ermöglicht“ (Wengeler 2007:181, Herv. i. O.) und Vertrauen und Misstrauen gegenüber Flüchtlingen vor allem implizit/ latent konstruiert wird.

11 S. oben zum Forschungsstand sowie zum Zusammenhang zwischen der Phraseologie und der Diskurslinguistik Stumpf \& Kreuz (2016) sowie Stumpf \& Kreuz (2018), vgl. auch Fußnote 13. 
oder Schlüsselwörter erfolgt. In diesem Beitrag wird aber nur die Funktion der formelhaften Wendungen als sprachliche Realisierungsmittel solcher Topoi näher betrachtet, mit denen vor dem Hintergrund der Argumentation contra Flüchtlingsaufnahme Ängste mehr oder weniger implizit konstruiert werden. Im untersuchten Korpus (siehe zur Datengrundlage weiter unten) wurden insgesamt 16 formelhafte Wendungen (types) dieses Typs induktiv ermittelt. Im Kapitel 4 werden drei formelhafte Wendungen exemplarisch herausgegriffen und näher untersucht. Filatkina (2018a: 164) definiert formelhafte Wendungen als

\begin{abstract}
Einwortausdrücke, typologisch heterogene Kombinationen aus mehreren Konstituenten bzw. ganze Sätze und/oder Texte, die holistisch verstanden werden müssen, sich auf unterschiedlichen (auch noch nicht abgeschlossenen) Stadien der formalen, semantischen und funktionalen Konventionalisierung befinden, auf Gebrauchskonventionen einer Sprachgemeinschaft beruhen, deren etablierte kulturelle (auch kommunikative) Erfahrungen und Wissensbestände sie tradieren, und die sich durch eine starke Funktionalisierung im Kommunikationsprozess bzw. im Textaufbau auszeichnen können.
\end{abstract}

Der Begriff formelhafte Wendung eignet sich besser zur Bezeichnung der untersuchten Analyseeinheit als der Terminus Phraseologismus, weil es sich bei den im Korpus vorkommenden Mehrworteinheiten nicht um Phraseologismen im engeren Sinne handelt ${ }^{12}$. Es liegen stattdessen heterogene Kombinationen (in der Regel) aus mehreren Konstituenten vor, die im Hinblick auf ihre diskursive Funktion das kollektive Denken, Fühlen und Wollen (bzw. Mentalitäten nach Hermanns 1995) nicht nur tradieren bzw. nicht nur eine wissenskondensierende, sondern auch eine wissenskonstruierende Funktion haben, indem sie auch auf die Veränderungen in der Konstruktion des kollektiven Wissens verweisen (vgl. dazu Stumpf \& Kreuz 2016: 16-17) ${ }^{13}$. Damit werden die Gemeinsamkeiten zwischen der Diskurslinguistik und der Phraseologie im weitesten Sinne in den Blick genommen. ${ }^{14}$

12 Somit wird die These von Stumpf (2017) bestätigt, demzufolge sich die Aufteilung in phraseologischen Kern und Peripherie für die innerdisziplinäre Anbindung der Phraseologie (wie etwa an die Diskurslinguistik) als „Hemmschuh“ (Stumpf 2017: 79-80) erweist.

13 Methodische Überlegungen zur diskurslinguistischen Untersuchung von Phraseologismen finden sich in Stumpf \& Kreuz (2016) sowie in Römer (2018). Römer (2018) diskutiert die Zusammenhänge zwischen der Phraseologieforschung und der Argumentationsanalyse. Zu den argumentativen Funktionen von Phraseologismen auf der Textebene vgl. Wirrer (1998), Pfeiffer (2016) und Lüger (2018).

14 Vgl. dazu die Beiträge in Stumpf \& Filatkina (2018a). Beide Disziplinen lassen sich der „Formelhafte Sprache“-Forschung zuordnen, die davon ausgeht, dass Formelhaftigkeit „ein zentrales Organisationsprinzip des sprachlichen Könnens - und nicht ein Randphänomen oder eine Ausnahme darstellt“ (Filatkina 2018a: 56) und nach musterhaften sprachlichen Strukturen 
Die Korpusanalyse hat gezeigt, dass Migrationsängste zwar einen wichtigen Aspekt bei der sprachlichen Konstruktion des (fehlenden) Vertrauens gegenüber Flüchtlingen darstellen. Andererseits spielen sie nicht in allen Vertrauensindikatoren gleichermaßen eine Rolle. So ergibt sich aus der Korpusanalyse, dass die Angst vor Flüchtlingen vor allem innerhalb des Vertrauensindikators Zukunftserwartungen zentral ist und dabei häufig im Zusammenhang mit Misstrauen vorkommt. Daher konzentrieren sich die nachfolgenden Ausführungen nur auf Zukunftserwartungen. Das bedeutet aber nicht, dass Migrationsängste in anderen Indikatoren überhaupt nicht konstruiert werden oder dass keine anderen sprachlichen Mittel zur Angstkonstruktion im Korpus vorkommen. ${ }^{15}$ Wie bei der Darstellung der Analyseergebnisse gezeigt wird, überschneiden sich sowohl die Vertrauensindikatoren als auch die einzelnen sprachlichen Mechanismen der Angstkonstruktion.

Die Auswahl der im nächsten Kapitel untersuchten formelhaften Wendungen richtet sich nicht nach der Häufigkeit ihres Vorkommens im Korpus. Vielmehr spielt einerseits ihre diskursübergreifende Verwendung in verschiedenen Diskursen und Textsorten (wie etwa in Karikaturen bei die Stimmung kippt), andererseits aber die Tatsache, dass manche im Flüchtlingsdiskurs entstanden sind (wie etwa die Grenze der Aufnahmefähigkeit) eine entscheidende Rolle. Darüber hinaus ergibt sich die angstkonstruierende Funktion erst aus einem bestimmten diskursiven Kontext. ${ }^{16}$ Deswegen ist nicht die formelhafte Wendung an sich für die Bestimmung der diskursiven Funktion entscheidend, sondern der Kontext, in dem sie vorkommt.

Der Analyse liegt ein manuell zusammengestelltes Korpus von rund 6000 Texten zugrunde, die den folgenden überregionalen Tages- und Wochenzeitungen entnommen sind: Frankfurter Allgemeine Zeitung (FAZ), Tageszeitung

sucht (vgl. Stumpf \& Filatkina 2018b: 3). In diesem Sinne sollten idealerweise sprachliche Muster in ihrer Gesamtheit sowie im Hinblick auf das Zusammenwirken des Formelhaften und der Variation (vgl. Filatkina 2018a: 3) erschlossen werden.

15 Vgl. etwa die Angstkonstruktion durch die Zuschreibung religiöser Andersartigkeit Die Flüchtlinge kommen aus Staaten, die vom Islam geprägt sind innerhalb des Indikators Vertrauenswürdigkeit im folgenden Korpusbeleg: „Zur Ehrlichkeit gehört auch die banale Feststellung, dass eben nicht eine Million Dänen, Franzosen oder Russen kommen. Die Flüchtlinge kommen aus Staaten, die vom Islam geprägt sind.“ (FAZ 13.09.2015, „Es gilt das Grundgesetz“). 16 Vgl. dazu Filatkina (2018b: 35) bezüglich der formelhaften Wendung Wir schaffen das: „However, the crucial factor in the emergence of this formulaic pattern is not the frequency of use as such but its origin in the refugee discourse and the acute and controversially discussed importance of this discourse for German political and everyday life“. Ähnliches gilt laut Filatkina für Make America great again, fake news und war on terror. 
(taz), Süddeutsche Zeitung (SZ), Spiegel, Zeit, Spiegel Online (SPON), Zeit Online (ZON) und Frankfurter Rundschau (FR). Die Texte wurden manuell aus den elektronischen Datenbanken ${ }^{17}$ bzw. aus der Datenbank LexisNexis ${ }^{18}$ anhand des Suchstrings ${ }^{*}$ flucht ${ }^{*} O R{ }^{*} f l u ̈ c h{ }^{*} O R{ }^{*}{ }^{*}$ igra ${ }^{*}$ erhoben, wobei der thematische Bezug zur jüngsten Flüchtlingsdebatte ein zentrales Kriterium für die Aufnahme der Zeitungstexte in das Korpus darstellt. Der Untersuchungszeitraum umfasst die Zeit zwischen der Aussage der Bundeskanzlerin Angela Merkel Wir schaffen das auf der Bundespressekonferenz am 31.08.2015 und 01.02.2017 (Terroranschlag in Berlin am 19.12.2016 und die darauffolgende Berichterstattung sowie der Jahrestag der Kölner Silvesternacht). Nachdem die oben erwähnten Vertrauensindikatoren annotiert wurden und die Toposanalyse durchgeführt wurde, wurde das Korpus auf rund 2000 Texte reduziert, weil die Vertrauensindikatoren nicht in allen 6000 Texten ausfindig gemacht werden konnten und die Toposanalyse nicht bei allen mit Indikatoren annotierten Textstellen durchführbar war. Diese Textmenge stellt die Grundlage für die Analyse dar.

\section{Analyse}

Die Korpusanalyse hat ergeben, dass innerhalb des Indikators Zukunftserwartungen zwei Formen von angstbesetzten Zukunftserwartungen vorkommen: zum einen Ängste vor dem Verlust des Gewohnten (die Angst vor kulturellen Veränderungen, vor Überfremdung, vor Konkurrenz um Arbeitsplätze sowie die sicher-heitsbezogenen Ängste wie etwa die Terrorangst oder die Angst vor dem Anstieg der Kriminalität durch Flüchtlinge) und zum anderen die Angst vor der finanziellen Belastung Deutschlands angesichts der hohen Kosten der Flüchtlingsaufnahme- und integration.

Diese linguistisch ermittelte Aufteilung stimmt teilweise mit den Formen der Migrationsangst überein, die in der sozialwissenschaftlichen Migrationsforschung erwähnt werden (vgl. Foroutan 2016 oder Bade 2016). Laut Foroutan (2016) sind im Migrationskontext soziale und nationale Ängste voneinander zu unterscheiden. Während nationale Ängste auf dem Bekenntnis zu nationaler Identität und gleichzeitiger Abwehr von Minderheiten beruhen (vgl. Foroutan 2016: 97), umfassen soziale Ängste die Angst vor sozialem Abstieg und vor jeder

17 Es handelt sich um folgende Datenbanken: Das FAZ-Bibliotheksportal: https://www.fazbiblionet.de/faz-portal sowie das Volltext-Archiv der Süddeutschen Zeitung https://archiv. szarchiv.de/Portal/restricted/HomeExtendedResultList.act (12.05.2019).

18 https://www.nexis.com/ (12.05.2019). 
Art der sozialen Verunsicherung, die auf der Zunahme struktureller Ungleichheit fußt (vgl. Foroutan 2016: 100).

Nachfolgend werden drei formelhafte Wendungen unter Berücksichtigung des diskursiven Kontextes und ihrer Varianten ${ }^{19}$ exemplarisch analysiert. Es handelt sich um folgende formelhafte Wendungen: Die Stimmung kippt, Deutschland verändert sich und die Grenze der Aufnahmefähigkeit, ${ }^{20}$ die zur Konstruktion von düsteren Zukunftsprognosen, der Kultur- und Konkurrenzängsten und der Angst vor (finanzieller) Belastung durch Flüchtlinge eingesetzt werden.

\subsection{Düstere Zukunftsprognosen}

Zunächst wird auf die sprachliche Konstruktion von angstbesetzten Folgen der Flüchtlingsaufnahme durch die formelhafte Wendung die Stimmung kippt eingegangen. Sie ist im Unterschied etwa zu Wir schaffen das nicht im aktuellen Flüchtlingsdiskurs entstanden. Außerdem ist sie nicht nur für diesen Diskurs typisch. Im Flüchtlingsdiskurs findet sie sich auch in Karikaturen ${ }^{21}$ sowie in den Zeitungsüberschriften:

(3) Der Nürnberger Oberbürgermeister Ulrich Maly (SPD) äußerte, die Kommunen lebten im Widerspruch zwischen dem deutschen Asylrecht ohne Obergrenzen und den begrenzten „Möglichkeiten vor Ort“. Darauf gebe es keine leichten Antworten. Die Stimmung an der SPD-Basis beschrieb er ebenso wie der Oberbürgermeister Gelsenkirchens, Frank Baranowski - als geteilt: Es gebe immer noch viele, die für die Willkommenskultur stünden. Es gebe aber auch Ängste. In der nicht-öffentlichen Diskussion äußerten Kommunalpolitiker die Sorge darüber, was nach der ersten Hilfe komme, wenn die Integration anfange. Wenn etwa Schulklassen vergrößert würden, könnte die Stimmung kippen.

(FAZ 13.11.2015 „Gabriel: Tempo der Einwanderung reduzieren“)

19 Vgl. dazu Burger (2015: 22-25).

20 Auch anderen formelhaften Wendungen wie etwa der in mehreren empirischen Untersuchungen analysierten Wendung Wir schaffen das (vgl. Fußnote 7) kann in bestimmten Kontexten eine angstkonstruierende Rolle zugewiesen werden. Im Rahmen dieser Analyse werden sie jedoch aus Platzgründen nicht behandelt.

21 Vgl. https://www.stuttmann-karikaturen.de/karikaturen/2016/stimmung_kippt_kol_b.jpg (12.05.2019). 
(4) Der CDU-Fraktionsvorsitzende im niedersächsischen Landtag, Björn Thümler, spricht davon, dass ,die Stimmung in der Bevölkerung vielerorts gekippt“ sei. „Die Unterbringungskapazitäten im Land sind nahezu erschöpft“, sagt er. „Aus der anfänglichen Solidarität droht vielerorts Ablehnung zu werden.“ (DER SPIEGEL 02.10.2015, „An der Grenze“)

Innerhalb der Argumentation contra Flüchtlinge wird sie etwa von dem Bundesrichter Thomas Fischer, dem Präsidenten des ifo Instituts Hans-Werner Sinn, verschiedenen Lokalpolitiker`innen (vgl. Beleg (4)), die über die Belastung ihrer Gemeinden klagen, dem damaligen Vizekanzler Sigmar Gabriel sowie von den Flüchtlingshelfer*innen bei der Konstruktion der Angst vor dem Zuwachs fremdenfeindlicher Ausschreitungen oder Wirtschaftsforscher*innen, die vor den Konkurrenzkämpfen warnen oder von dem Vorstandsvorsitzenden der Jüdischen Gemeinde Salomon Korn verwendet.

Die diskursive Funktion der Angstkonstruktion erfüllt sie zumeist als sprachliches Realisierungsmittel des Topos der düsteren Zukunfts- und Gegenwartsdiagnose, dem folgendes Muster zugrunde liegt: Weil die Flüchtlingsaufnahme mit schlimmen Konsequenzen verbunden ist, sind die Flüchtlinge nicht aufzunehmen. ${ }^{22}$ Mit dieser formelhaften Wendung wird die Angst vor den Folgen der absteigenden Aufnahmebereitschaft wie etwa vor dem Anstieg der Fremdenfeindlichkeit o. Ä. zum Ausdruck gebracht. Deswegen kommt sie auch im Kontext der Kölner Nacht und im Zusammenhang mit der Thematisierung der Fremdenfeindlichkeit vor. Bei den Migrationsängsten, die mit diesem Topos konstruiert werden, handelt es sich ganz um die Angst vor künftiger Entwicklung der Flüchtlingsintegration, die aber oft mit der Konstruktion von Konkurrenzkämpfen und Belastungen vermischt wird wie etwa in Beleg (3) mit den Konkurrenzängsten und in Beleg (4) mit dem Belastungstopos (s. dazu unten).

In Beleg (3) werden Kommunalpolitikerinnen als angstschürende Diskursakteur`innen zitiert. Die Diskursakteurin/der Diskursakteur spricht von einer Zeit der Willkommenskultur und einer Zeit danach, in der die Schwierigkeiten der Integration beginnen und die angesichts der Sorge um die Knappheit der Ressourcen (vgl. wenn etwa Schulklassen vergrößert werden) zur negativen Stimmung in der Bevölkerung führen könnte. Auch das Modalverb könnte trägt zur Konstruktion der düsteren Zukunftsprognose bei, indem es auf ungewisse Zukunft verweist. In diesem Kontext werden sowohl die bevorstehenden Integrationsprobleme als auch die Verteilung von Ressourcen als negative Folgen problematisiert. Genauer gesagt lässt sich die Angst vor der Konkurrenz mit den

22 Vgl. zu diesem Topos in Krisendiskursen Römer (2017). 
Flüchtlingen, die durch könnte die Stimmung kippen konstruiert wird, nicht getrennt von den düsteren Zukunftsszenarien im vorherigen Satz betrachten. Diese formelhafte Wendung kommt häufig im Zusammenhang mit den expliziten sprachlichen Mitteln der Angstkonstruktion vor wie beispielsweise mit den Lexemen aus dem lexikalischen Feld Angst (vgl. Sorge in (3) sowie mit dem Verb drohen (vgl. Beleg (4)). Zudem fungiert der Tempuswechsel vom Präsens zum Perfekt (dass die Stimmung (...) gekippt sei) in Beleg (4) als sprachliches Realisierungsmittel des Topos der düsteren Gegenwartsdiagnose, mit dem die negativen Folgen sich bereits in der Gegenwart abzeichnen (vgl. Römer 2017: 164). Die formelhafte Wendung in Beleg (4) ist aber für die Angstkonstruktion nicht zentral. Erst durch zwei nachfolgende Sätze, in denen der Belastungstopos (Die Unterbringungskapazitäten im Land sind nahezu erschöpft) und die Konstruktion einer Zeit der Solidarität und einer darauffolgenden Zeit der Ablehnung erfolgt, wird sie inhaltlich näher bestimmt. Die Angstkonstruktion innerhalb des Topos der düsteren Zukunfts- bzw. Gegenwartsdiagnose ist daher eher von den anderen sprachlichen Mitteln abhängig, und zwar insbesondere von den Zeitangaben (vgl. anfänglich bzw. Solidarität versus Ablehnung in Beispiel (4)).

Ähnlich wird der Topos der düsteren Zukunftsprognose innerhalb einer weiteren Variante des Topos (vgl. Belege (5) und (6)) maßgeblich durch Die Stimmung kippt sprachlich realisiert, indem die Angst vor dem Zuwachs der Fremdenfeindlichkeit konstruiert wird:

(5) Ja, ich sehe den Flüchtlingsgipfel als Erfolg: Der Konsens reicht von Ramelow bis Seehofer, mittendrin die Kanzlerin. Damit ist ein wichtiges Signal ausgesandt, dass alle Parteien in Regierungsverantwortung in der Krise zusammenstehen. Wenn das nicht mehr so ist, dann kippt die Stimmung in der Bevölkerung. Und es ist auch ein Signal an Europa. Stellen Sie sich vor, die AfD käme in die Größenordnung von Front National oder Ukip, da wäre doch Panik bei uns. (Winfried Kretschmann in: taz 17.10.2015, „Mir brennt hier wirklich jeden Tag der Kittel“)

(6) Aber er [Sigmar Gabriel] mahnt auch Realismus an: Die Integration werde nicht problemlos ablaufen, Reibereien im Alltag seien absehbar. Da ihn die Sorge umtreibt, die Stimmung im Lande könne irgendwann kippen, sagt er noch: „Wir müssen das Land zusammenhalten.“

(FAZ 20.01.2016, „Das Geschwätz von gestern“)

Darüber hinaus steht die negative Zukunftsprognose im Zusammenhang mit der Erwartungshaltung an die Regierung. In den Augen des Diskursakteurs muss 
die Regierung die Handlungsfähigkeit zeigen, indem sie bestimmte Maßnahmen ergreift, um die negative Zukunftsprognose zu verhindern (Topos der Handlungsfähigkeit). Der Diskursakteur (vgl. Beleg (5)) konstruiert anhand der formelhaften Wendung die Angst vor den Folgen einer uneinheitlichen Flüchtlingspolitik und entwirft gleichzeitig ein Szenario, in dem die rechten Parteien die Oberhand gewinnen. Die formelhafte Wendung ist in den Konditionalsatz eingebettet, sodass die Ursache des Stimmungsumschwungs explizit genannt wird. Diese düstere Zukunftsprognose wird durch die nachfolgenden Sätze verstärkt, in denen die Angst vor dem Erfolg rechtspopulistischer Parteien konstruiert wird.

Die Aussage von Sigmar Gabriel in Beleg (6) steht im Zusammenhang mit der Kölner Nacht und enthält eine Erwartungshaltung (vgl. „Wir müssen das Land zusammenhalten"), die sich auf die Maßnahmen gegen die negativen Folgen der Flüchtlingsaufnahme bezieht. Die formelhafte Wendung taucht als indirektes Zitat auf und wird zur Konstruktion von negativen Szenarien eingesetzt, die irgend-wann in der Zukunft auftreten können. Die Expansion um irgendwann verstärkt die Ungewissheit bezüglich der Frage, wann die düstere Prognose eintreten könnte. Die Aneinanderreihung von düsteren Zukunftsszenarien in zwei aufeinander folgenden parataktischen Sätzen („Die Integration werde nicht problemlos ablaufen, Reibereien im Alltag sind absehbar“) ist zwar für die sprachliche Konstruktion des Topos der düsteren Zukunftsprognose zentral, aber durch Die Stimmung kippt wird implizit ein zusätzlicher Aspekt ins Spiel gebracht, und zwar die Angst vor dem Zuwachs der Fremdenfeindlichkeit, die direkt mit der Erwartungshaltung Wir müssen das Land zusammenhalten verbunden ist.

In Beispiel (7) folgt der Topos der düsteren Zukunftsprognose auf den Belastungstopos:

(7) Wenn die Erstaufnahmeeinrichtungen weiter überlastet sind, wenn die Flüchtlinge und Asylbewerber im Winter kein Dach über dem Kopf haben, wenn Gemeinden sich im Stich gelassen sehen, dann kann die Stimmung in Deutschland schnell kippen: weg vom freundlichen Willkommen hin $\mathrm{zu}$ Überdruss, Aggression und Ausschreitungen. Die ohnehin erschreckend rasch gewachsene Zahl von Übergriffen, weit mehr als 300 in diesem Jahr, könnte schneller steigen. (FAZ 06.09.2015, „Die Geschichten vom guten Flüchtling“)

Die formelhafte Wendung verbindet die beiden Topoi und wird dadurch inhaltlich näher bestimmt. Die befürchteten Folgen beziehen sich auf die Angst vor 
dem Zuwachs der fremdenfeindlichen Stimmung in der Bevölkerung und werden hauptsächlich durch Kontraste auf lexikalischer Ebene (Willkommen versus Überdruss, Aggression, Ausschreitungen) konstruiert.

In einigen Korpusbelegen wird durch die Stimmung kippt eine distanzkritische Stellung zu den angstbesetzten Zukunftsprognosen genommen (vgl. Beleg (8)):

(8) Aber die Stimmung kippt nicht von allein, sie wird gekippt. Wer - außer den inzwischen tatsächlich oftmals sehr erschöpften HelferInnen - hat denn tatsächlich durch die Ankunft Geflüchteter eine Veränderung in seinem Leben erfahren? Okay, die eine oder andere Turnhalle steht vorübergehend nicht mehr für Schul- und Vereinssport zur Verfügung. Aber sonst? Es ist ja kein Zufall, dass die Rechten in aller Regel mit konstruierten oder schlicht erfundenen Horrorgeschichten operieren müssen, um die Ablehnung zu erzeugen, die sie wollen. Und Focus Online, Horst Seehofer und mitunter der Bundesinnenminister köcheln mit. (taz 25.11.2015, „Wir schaffen das! Oder nicht?")

Die Medien und/oder rechte Politiker^innen werden als angstschürende Instanzen kritisiert, indem ihnen die Instrumentalisierung von Ängsten vorgeworfen wird. Inwieweit die Diskursakteurin/der Diskursakteur dabei auf den Angstabbau abzielen, ist stark kontextabhängig. In Beleg (8) wird die formelhafte Wendung durch nicht von allein sowie durch die Passivform verändert, um die düsteren Zukunftsprognosen als das Produkt bestimmter Diskursakteur*innen zu konstruieren bzw. sie als schlicht erfundene Horrorgeschichten abzuwerten.

\subsection{Kultur- und Konkurrenzängste}

In diesem Kapitel steht die formelhafte Wendung Deutschland verändert sich im Vordergrund, die hauptsächlich (aber nicht ausschließlich) zur Konstruktion von Ängsten vor dem Verlust des Gewohnten eingesetzt wird. Sie fungiert vor allem als sprachliches Realisierungsmittel des Topos der kulturellen Veränderungen (Weil Flüchtlinge eine andere Mentalität haben und Deutschland in kultureller/religiöser Hinsicht zu verändern drohen, sollten sie nicht aufgenommen werden). sowie des Konkurrenztopos (Weil die Gefahr besteht, dass Flüchtlinge und Einheimische um Arbeitsplätze, Wohnraum usw. konkurrieren, sollten die Flüchtlinge nicht aufgenommen werden). 
Die formelhafte Wendung Deutschland verändert sich wird innerhalb der Diskurspositionen $^{23}$ contra Flüchtlinge meistens von den Politiker*innen und Expert*innen gebraucht und finden sich daher in den Aussagen des Innenministers Thomas de Maizière, des CSU-Politikers Markus Söder, des Direktors des Max-Plank Instituts zur Erforschung multireligiöser und multiethnischer Gesellschaften Steven Vertovec, der Politikwissenschaftlerin und Direktorin des John Stuart Mill Instituts für Freiheitsforschung in Heidelberg Ulrike Ackermann, des Innenministers Saarlands Klaus Bouillon (CDU) oder der CDU-Bundestagsabgeordneten Erika Steinbach sowie in einem Kommentar von Boris Palmer und Jens Sphan in der FAZ vom 02.09.2015: ${ }^{24}$

(9) Die Zuwanderung Hunderttausender Menschen aus anderen Kulturkreisen und Religionen wird die kulturelle Statik unseres Landes verändern. Es werden in diesem Jahr mehr Menschen einwandern als Kinder hier geboren werden. Deutschland verändert sich. (Markus Söder in: FAZ 10.09.2015, „Das Asylrecht muss eingeschränkt werden“)

(10) Ich zähle zu denen, die davon überzeugt sind, dass die Integration der nach Deutschland strömenden Flüchtlingsmassen eine naive Wunschvorstellung darstellt und die unbegrenzte Zuwanderung von Menschen muslimischer Religionsangehörigkeit Deutschland nachhaltig zu verändern droht. (Leserbriefschreiber in: FAZ 02.12.2015, „Die rechtstreue Mitte ohne politische Heimat“)

In Beispiel (9) fungiert Deutschland verändert sich als Realisierungsmittel des Topos der kulturellen Veränderungen. Indem die Komponente Deutschland durch die kulturelle Statik unseres Landes ersetzt wird, werden die kulturellen Unterschiede zwischen den Einheimischen und den Flüchtlingen betont. Außerdem trägt die Wiederholung von kulturell im Zusammenhang mit der stereotypen Zuschreibung Menschen aus anderen Kulturkreisen zur Verstärkung von Überfremdungsängsten bei. Das Überfremdungsszenario bezieht sich auf die

23 Darunter wird die (ideologische) Einstellung der Diskursakteurin/ des Diskursakteurs verstanden.

24 Zur Verwendung der formelhaften Wendung in einem nicht angstschürenden Kontext vgl. die Aussage der Grünen-Politikerin Katrin Göring- Eckard in einer Rede auf dem Parteitag der Grünen am 20.11.2015: „Und die Fraktionsvorsitzende Katrin Göring-Eckardt, die auf der griechischen Insel Lesbos ein Flüchtlingsboot in Empfang nahm, sagt: ,Unser Land wird sich ändern, und zwar drastisch. Und ich freue mich drauf!““ (SPON 21.11.2015, „... denn sie wissen nicht, was sie wollen“). 
nahe Zukunft, indem der Diskursakteur eine bald eintretende demografische Verän-derung bzw. einen Völkeraustausch voraussagt. In der Aussage von Markus Söder wird das Lexem verändern wiederholt, sodass die befürchtete kulturelle Veränderung bereits sichtbar ist. Auch der Leserbriefschreiber in Beleg (10) verstärkt durch die unbegrenzte Zuwanderung von Menschen muslimischer Religionsangehörigkeit die angstkonstruierende Funktion von etwas verändert Deutschland. Das Überfremdungsszenario wird außerdem durch Stereotype sowie durch die Lexeme nachhaltig und drohen konstruiert.

Wie ersichtlich, wird die diskursive Funktion von Deutschland verändert sich erst durch das Zusammenspiel mit anderen sprachlichen Mitteln deutlich wie etwa durch Gruppenbezeichnungen Menschen muslimischer Religionsangehörigkeit oder Menschen aus anderen Kulturkreisen und Religionen.

Während mit dem Topos der kulturellen Veränderungen die Kulturangst bzw. „Angst vor einer Gefährdung der eigenen Wertvorstellungen und Lebensformen oder dessen, was man dafür hält, durch als fremd empfundene Wertvorstellungen und Lebensformen oder das, was man dafür hält“" (Bade 2016: 580) konstruiert wird, stehen im Vordergrund des Konkurrenztopos die Ängste um die Verteilung von Ressourcen oder sogenannte Konkurrenzängste, die verschiedene Formen sozialer Verunsicherung (wie unter anderem Angst vor sozialem Abstieg, Konkurrenz um Arbeitsplätze, billige Wohnungen, Kitaplätze usw.) umfassen:

(11) Um die Migranten $\mathrm{zu}$ integrieren, wird sich diese Gesellschaft verändern müssen. Wir werden Verteilungskämpfe erleben, um billigen Wohnraum zum Beispiel, um schlecht bezahlte Jobs oder kostenlose Gesundheitsvorsorge (DIE ZEIT 17.09.2015, „Starke Truppe“)

(12) Die vielen Flüchtlinge werden die Stadt in vieler Hinsicht verändern. Noch mehr Menschen werden sich um die ohnehin raren günstigen Wohnungen reißen, auch die Konkurrenz auf dem Arbeitsmarkt, gerade dem für einfache Tätigkeiten, wird wohl zunehmen. (taz 02.01.2016, „Ein Plan für die Neu-Berliner")

Die angstkonstruierende Funktion von Deutschland verändert sich innerhalb dieses Topos ist in Beispielen (11) und (12) im Vergleich zu den Belegen (9) und (10) nicht so stark ausgeprägt, weil die Konkurrenzangst hauptsächlich durch andere sprachliche Mittel bzw. vorwiegend auf lexikalischer Ebene (wie etwa durch Lexeme Verteilungskämpfe, Konkurrenz auf dem Arbeitsmarkt) konstruiert wird. Daher haben die Varianten von Deutschland verändert sich in Belegen (11) 
und (12) die Funktion die sprachliche Konstruktion des Topos zu signalisieren oder zu stützen.

\subsection{Belastungsszenarien}

Die Angst vor finanziellen Risiken und der Überforderung der Gesellschaft durch die Flüchtlingsaufnahme steht im Vordergrund der Zukunftserwartungen, deren sprachliche Konstruktion nachfolgend anhand der formelhaften Wendung die Grenze der Aufnahmefähigkeit skizziert wird.

Mit dieser formelhaften Wendung konstruieren die Diskursakteur`innen hauptsächlich im Rahmen des Belastungstopos ${ }^{25}$ die Sorge, dass Deutschland sich wegen fehlender Aufnahmekapazitäten, finanzieller Mittel u. Ä. mit der Aufnahme von Flüchtlingen überfordert. Sie kommt zunehmend im Kontext der Debatte um Obergrenzen (ab Herbst 2015) vor, wird aber von Thomas de Maizière schon Anfang September 2015 gebraucht. Auch der CDU-Politiker Stefan Grünert spricht Anfang September 2015 davon, dass die Bundesländer angesichts der Entscheidung der Kanzlerin die Flüchtlinge aus Ungarn aufzunehmen an den Grenzen ihrer Kapazitäten gelangt seien (vgl. FAZ 07.09.2015). Ähnliche Formulierung (Grenzen der Aufnahmekapazität) findet sich in den Aussagen von Thomas Oppermann (SPD), während der damalige Bürgermeister Hamburgs und SPD-Politiker Olaf Scholz gleichzeitig die Grenzen der Belastbarkeit erwähnt und für die Trennung von schutzbedürftigen und nicht schutzbedürftigen Flüchtlingen plädiert. Ähnlich äußern sich Anfang September 2015 auch Udo di Fabio und der hessische Ministerpräsident Volker Bouffier. Auch der Bundespräsident Joachim Gauck spricht davon, dass unsere Aufnahmekapazität begrenzt sei ${ }^{26}$. In einem Spiegel-Interview prognostiziert Sigmar Gabriel Anfang Oktober 2015, dass die Zahl der aufzunehmenden Menschen auf eine Million steigen wird (,Wir nähern uns in Deutschland mit rasanter Geschwindigkeit den Grenzen unserer Möglichkeiten“ SPON 02.10.2015) und formuliert eine ähnliche Aussage im ZDF-Sommerinterview Ende August 2016. Die Aussage Gabriels wird mehrfach kritisch aufgegriffen (vgl. dazu Beispiel (18)). Im Unterschied zu den Politikern fokussiert sich der Leiter der Heinrich-Böll-Stiftung Ralf Fücks auf „die Grenzen der kulturellen Integrationsfähigkeit der Flüchtlinge“ (SPON

25 Diesem Topos liegt folgendes Muster zugrunde: Weil die Aufnahme von Flüchtlingen hohe finanzielle Risiken mit sich bringt, sind die Flüchtlinge nicht aufzunehmen.

26 Vgl. dazu: https://www.spiegel.de/politik/deutschland/gauck-rede-bundespraesident-sprichtueber-fluechtlingskrise-a-1054984.html (12.05.2019). 
28.10.2015) und bringt sie in Verbindung mit der Aufnahmebereitschaft. Im Zusammenhang mit der Debatte um die Obergrenzen ist von den Grenzen unseres Integrationspotentials (Reiner Haseloff, CDU) die Rede. Dennoch überwiegt die angstkonstruierende Funktion der formelhaften Wendung hinsichtlich der Aufnahmekapazitäten und finanziellen Belastungen ${ }^{27}$. Im Zusammenhang mit der Debatte um die Familienzusammenführung und den subsidiären Schutz spricht der innenpolitische Sprecher der Unionsfraktion im Bundestag Stephan Mayer von den Grenzen der Möglichkeiten unseres Landes und konstruiert damit den Belastungstopos: „Wir haben den Nachzug für subsidiär Schutzberechtigte nicht aus Hartherzigkeit eingeschränkt, sondern aus Einsicht in die Grenzen der Möglichkeiten unseres Landes“, sagte Mayer dieser Zeitung. (FAZ 27.08.2016. „Lieber Bürgerkrieg als Deutschland“).

Die Belastung bezieht sich hauptsächlich auf die Überforderung des Landes im Allgemeinen wie etwa auf die Unterbringungskapazitäten. In vielen Fällen sind es einzelne Städte und Gemeinden, die angesichts der bevorstehenden Flüchtlingsaufnahme an die Grenzen ihrer Kapazitäten (FAZ 20.02.2016) kommen. So ist etwa im Kontext des im Auftrag gegebenen Gutachtens von Udo di Fabio (Januar 2016) auch von den Grenzen der Funktionsfähigkeit Bayerns die Rede. Gemeint sind dabei fast ausnahmslos quantitative Aspekte wie etwa die Zahl der aufzunehmenden Menschen, während Überforderung angesichts der schwierigen Integration (vgl. dazu oben die Aussagen von Reiner Haseloff und Ralf Fücks) seltener konstruiert wird:

(13) Thomas de Maizière: Das politische Asyl kennt natürlich aus guten Gründen keine Obergrenze. Aber im Normalfall gibt es Obergrenzen der Aufnahmefähigkeit eines Landes. Viele Experten sagen, dass für ein Land unserer Größenordnung eine Nettozuwanderung von 400000 bis 500000 Menschen schon ziemlich viel ist. (DIE ZEIT 03.09.2015, „Alles muss sehr schnell gehen“)

(14) Mit Familiennachzug werden aus einer Million Flüchtlingen drei oder vier und in den nächsten Jahren vielleicht sechs oder acht? Wo soll das enden? Welcher Politiker sagt uns, wohin die Reise geht in Deutschland, wie unser Land in zehn Jahren aussieht? Das Asylrecht kennt keine Obergrenze, wird wiederholt gesagt, aber die Aufnahmefähigkeit eines Landes hat eine sol-

27 Vgl. etwa die Aussage der Bayrischen Sozialministerin Emilia Müller: „Die bayerischen Bürger seien ,nicht an der Grenze unseres guten Willens angekommen, sondern an der Grenze unserer logistischen Möglichkeiten““ (SZ 18.01.2016, „Bayerisches Betreuungsgeld“). 
che. (Leserbriefschreiber in: SZ 28.01.2016, „Schützen und verteilen aber integrieren")

(15) Würde tatsächlich schon Integration betrieben, stünde schnell fest, dass Deutschland die Grenzen seiner Aufnahmefähigkeit längst überschritten hat. Besonders augenfällig ist das bei den Deutschkursen: Rechnet man die Zahl der Flüchtlinge und die nötigen Stunden hoch, die unterrichtet werden müssten, erreicht man eine Lehrerzahl, die jedes Maß sprengt - selbst wenn noch so viel Geld in die Integrationsmaßnahmen gepumpt wird. Ähnliches gilt für Integrationskurse, Kita-Ausbau und Ausbildungsplätze. (FAZ 03.02.2016, „Eine völlig unklare Aufgabe“)

In Beleg (13) wird die angstkonstruierende Funktion durch das Schlüsselwort Obergrenze verstärkt (vgl. ähnlich auch in (14)). In Beispiel (15) überschneidet sich der Belastungstopos, der durch die lexikalische Veränderung von Aufnahmefähigkeit zur Belastungsfähigkeit sprachlich realisiert wird, mit dem Topos der düsteren Gegenwartsdiagnose. Durch die Verbindung der formelhaften Wendung mit den Verben erreichen oder überschreiten wird die Konstruktion finanzieller Belastung hervorgehoben.

Ähnlich wie die Stimmung kippt und Deutschland verändert sich wird auch die Grenze der Aufnahmefähigkeit in kritisch-distanzierenden Diskurspositionen verwendet:

(16) Und was ist mit denen, die meinen in der Flüchtlingskrise stoße die deutsche Aufnahmebereitschaft und Aufnahmefähigkeit auf die Grenzen der „Realität“, wo doch der VW-Irrsinn belegt, dass kriminelle Energie und Dummheit keine natürlichen Grenzen kennt: Es sind Menschen, die Grenzen ziehen und sie überschreiten, nicht eine bequeme „Realität“. (taz 24.09.2015, „Die Volksrepublik“)

(17) Genau diese Stimmung nimmt die SPD-Spitze auf. Sie weiß, dass zu ihren Wählern auch Arbeiter oder arme Menschen gehören, die sich vor sozialer Konkurrenz durch die Flüchtlinge fürchten. Seit gut einer Woche betonen deshalb Spitzengenossen auffällig oft, dass Deutschland sich den Grenzen seiner Möglichkeiten nähere. (taz 13.10.2015, „Das liebenswerte Merkhofer“)

(18) Hätte der SPD-Chef offen über die Finanzierung des von ihm geforderten Zusammenhalts gesprochen, dann hätte das noch einen Vorteil gehabt: Die vagen Andeutungen über die Grenzen der Integrationskraft hätte er sich 
sparen können. Denn auch sie sind kein Naturgesetz, sondern ein Ergebnis von Politik. (FR 09.09.2016, „Der Preis der Integration“)

In den zitierten Belegen wird das Sprechen über die Grenzen der Integrationskraft, der Aufnahmekapazitäten oder der Aufnahmefähigkeit mehr oder weniger kritisch betrachtet, indem bestimmten sozialen Gruppen (vgl. Beleg (16)) bzw. Politiker*innen (vgl. Beispiele (17) und (18)) Ängste vor Überforderung des Landes durch die Flüchtlingsaufnahme zugeschrieben werden. In allen drei Beispielen positionieren sich die Diskursakteur*innen kritisch-distanzierend zu dem Belastungstopos, der einerseits durch die formelhafte Wendung sprachlich konstruiert wird, andererseits aber (insbesondere in (16) und (18)) negiert wird. Inwieweit die Diskursakteur`innen auf den Angstabbau abzielen, ist stark kontextabhängig. So steht die kritische Diskursposition insbesondere in (16) aber auch in (18) dem Angstabbau sehr nahe, während sie in (17) nicht so stark ausgeprägt ist.

\section{Fazit und Ausblick}

Dieser Beitrag setzte sich zum Ziel, die impliziten sprachlichen Mittel der Angstkonstruktion am Beispiel der formelhaften Wendungen aus diskurslinguistischer Sicht zu ermitteln und ihre diskursiven Funktionen zu erschließen. Dabei wurden drei formelhafte Wendungen in den Blick genommen und als sprachliche Realisierungsformen von Argumentationsmustern im aktuellen Flüchtlingsdiskurs betrachtet.

Abschließend lassen sich drei ineinandergreifende Aspekte unterscheiden: Die Funktion in Bezug auf die Frage, welche Migrationsängste konstruiert werden, die diskursive Funktion im Hinblick auf die Art und Weise der Angstkonstruktion und die Funktion der formelhaften Wendung als sprachliches Realisierungsmittel der Argumentationsmuster.

Im Hinblick auf die Art und Weise der Angstkonstruktion kann man festhalten, dass die angstkonstruierende Funktion von formelhaften Wendungen im Korpus zwei Aspekte umfasst, die als angstschürende und kritisch-distanzierende Funktion bezeichnet werden können. Während die angstschürende Funktion mehr oder weniger intendierte Instrumentalisierung von Ängsten umfasst, kommt die andere Funktion im distanz-kritischen bzw. neutral-vermittelnden Gebrauch zum Vorschein. Diese Unterscheidung ergibt sich aus der Frage Wer spricht? Dabei spielt der Indikator Diskursakteur*innen eine zentrale Rolle bzw. die Unterscheidung zwischen der Frage, ob eine Aussage einer Fremdinstanz in 
den Mund gelegt wird oder ob es sich tatsächlich um die Aussage dieser Fremdinstanz handelt. Grundsätzlich geht es um die Frage, ob die Angstkonstruktion als solche auf einer Metaebene konstruiert wird oder nicht. Genauer gesagt werden unter kritisch-distanzierender Funktion solche Korpusbelege verstanden, in denen die Diskursakteur`innen nicht selber Ängste konstruieren, sondern bestimmten sozialen Gruppen oder anderen Diskursakteur^innen die Instrumentalisierung von Ängsten zuschreiben oder sich davon distanzieren, indem sie das Schüren von Ängsten und Misstrauen mehr oder weniger kritisch betrachten. Die distanzierende bzw. relativierende Funktion lässt sich nach dem Modell von Radeiski (2011) nicht hundertprozentig der diskursiven Rolle Angstbewältigung zuordnen, weil es nicht immer um den Angstabbau geht. Demgegenüber ist die angstschürende diskursive Funktion als Angsterzeugung im Sinne von Radeiski (2011) zu verstehen. Darüber hinaus finden sich auch solche formelhaften Wendungen, die eindeutig eine angstbewältigende oder angstabbauende Funktion im Diskurs haben. Sie wurden aber nicht näher behandelt, weil dieser Aspekt den Rahmen des vorliegenden Beitrags sprengen würde. Da aber das Ziel der Diskurssemantik darin besteht, die konkurrierenden Wirklichkeitsauffassungen mit diskurslinguistischen Mitteln $\mathrm{zu}$ erschließen, sollte neben der für die Analyse zentralen angstschürenden oder angsterzeugenden auch die Funktion von formelhaften Wendungen bei der Distanzierung und kritischen Betrachtung der Instrumentalisierung von Ängsten nicht unerwähnt bleiben.

Die formelhaften Wendungen wurden ausschließlich als sprachliche Realisierungsmittel von Argumentationsmustern betrachtet, sodass ihnen in diesem Kontext unterschiedliche Funktionen zukommen, und zwar je nachdem, inwieweit sie im jeweiligen Kontext als zentrales sprachliches Realisierungsmittel eines Topos fungieren. So ist etwa die formelhafte Wendung Die Stimmung kippt in Beleg (4) nicht als zentrales sprachliches Realisierungsmittel bei der Konstruktion des Topos der düsteren Zukunftsprognose anzusehen, weil die düstere Zukunftsprognose hauptsächlich durch andere sprachliche Mittel wie etwa durch Zeitangaben und Kontraste auf lexikalischer Ebene inhaltlich näher bestimmt wird, während die Stimmung kippt die sprachliche Realisierung des Topos stützt bzw. signalisiert. Das lässt sich auch für Deutschland verändert sich bei der Konstruktion des Konkurrenztopos behaupten. Demgegenüber kann Deutschland verändert sich als zentrale Realisierungsform des Topos der kulturellen Veränderungen angesehen werden. Genauso werden hauptsächlich durch die Grenzen der Aufnahmekapazität (vorwiegend finanzielle) Belastungen vor dem Hintergrund des Belastungstopos konstruiert. Römer (2018: 151) nennt als eine der zentralen Funktionen von Phrasemen in Diskursen „die phraseolo- 
gische Stützung oder Realisierung von Argumentationen und die damit einhergehende Erzeugung von Plausibilitätseffekten, um bestimmte Ziele zu erreichen“. Auch die vorliegende Analyse zeigt, dass die untersuchten formelhaften Wendungen sowohl zur Stützung als auch zur Realisierung von Topoi im Flüchtlingsdiskurs eingesetzt werden. Es soll aber betont werden, dass diese Funktionen stark kontextabhängig sind.

Die Korpusanalyse hat gezeigt, dass anhand von formelhaften Wendungen je nach Kontext und Diskursakteur`in unterschiedliche Arten von Migrationsängsten konstruiert werden. Insbesondere bei Deutschland verändert sich ist in den Aussagen von Leserbriefschreiber ${ }^{\star}$ innen und manchen konservativen Politiker`innen auch die Konstruktion der Überfremdungsangst erkennbar. In inhaltlicher Hinsicht wird aber neben den Migrationsängsten, mit denen gegen die Aufnahme von Flüchtlingen argumentiert wird auch die Angst vor dem Rechtsruck bzw. dem Anstieg der Fremdenfeindlichkeit konstruiert (vgl. Belege (5) und (6)). Darüber hinaus finden sich auch distanzierend-kritische Aussagen zu den Migrationsängsten, mit denen die Ergebnisse in Kreußler \& Wengeler (2018) zumindest durch die vorliegende hermeneutische Analyse bestätigt werden konnten.

\section{Literatur}

Altheide, David L. (2002): Creating Fear: News and the Construction of Crisis. New York: De Gruyter.

Bade, Klaus (2016): Von Unworten zu Untaten: Kulturängste, Populismus und politische Feindbilder in der deutschen Migrations- und Asyldiskussion zwischen ,Gastarbeiterfrage ‘ und ,Flüchtlingskrise'. IMIS-Beiträge, 48/2016, 35-171. http://kjbade.de/wpcontent/uploads/2016/02/imis48.pdf (19.05.2019).

Bandelow, Borwin (2004): Das Angstbuch. Woher Ängste kommen und wie man sie bekämpfen kann. Reinbek bei Hamburg: Rowohlt.

Bauman, Zygmunt (2016): Die Angst vor den anderen. Ein Essay über Migration und Panikmache. Berlin: Suhrkamp.

Becker, Maria (2015): Der Asyldiskurs in Deutschland. Eine medienlinguistische Untersuchung von Pressetexten, Onlineforen und Polit-Talkshows. Frankfurt am Main: Peter Lang.

Belosevic, Milena (i. V.): Vertrauensgenerierung und Vertrauenserosion in der Flüchtlingsdebatte. 2015-2017. Eine diskurslinguistische Untersuchung der Argumentationsmuster. Dissertation. Universität Trier.

Bergenholtz, Henning (1980): Das Wortfeld „Angst“: Eine lexikographische Untersuchung mit Vorschlägen für ein großes interdisziplinäres Wörterbuch der deutschen Sprache. Stuttgart: Klett-Cotta. 
Bergmann, Jörg (2002): Paradoxien der Angstkommunikation - Über Veralten und Modernität der Angst. In: Mohammad E. Ardjomandi, Angelika Berghaus \& Werner Knauss (Hrsg.), Jahrbuch für Gruppenanalyse und ihre Anwendungen. Band 8: Der Andere in der GruppeAngst und Neugier, 1-15. Heidelberg: Mattes.

Böke, Karin (1997): Die Invasion aus den „Armenhäusern Europas“. Metaphern im Einwanderungsdiskurs. In: Matthias Jung, Martin Wengeler \& Karin Böke (Hrsg.), Die Sprache des Migrationsdiskurses. Das Reden über „Ausländer“ in Medien, Politik und Alltag, 164-193. Opladen: Westdeutscher.

Burger, Harald (2015): Phraseologie: Eine Einführung am Beispiel des Deutschen. 5. Aufl. Berlin: Schmidt.

Busse, Dietrich (1987): Historische Semantik. Analyse eines Programms. Stuttgart: Klett Cotta. Busse, Dietrich \& Wolfgang Teubert (1994): Ist Diskurs ein sprachwissenschaftliches Objekt? Zur Methodenfrage der historischen Semantik. In: Dietrich Busse, Fritz Hermanns \& Wolfgang Teubert (Hrsg.), Begriffsgeschichte und Diskursgeschichte. Methodenfragen und Forschungsergebnisse der historischen Semantik, 10-28. Opladen: Westdeutscher.

Fábián, Annamária (2019): „Wir schaffen das!“. In: Annamária Fábián \& Igor Trost (Hrsg.), Sprachgebrauch in der Politik. Grammatische, lexikalische, pragmatische, kulturelle und dialektologische Perspektiven, 77-102. Berlin/Boston: De Gruyter.

Filatkina, Natalia (2018a): Historische formelhafte Sprache. Theoretische Grundlagen und methodische Herausforderungen. Berlin/Boston: De Gruyter.

Filatkina, Natalia (2018b): Expanding the Lexicon through Formulaic Patterns: The Emergence of Formulaicity in Language History and Modern Language Use. In: Sabine Arndt-Lappe, Angelika Braun, Claudine Moulin \& Esme Winter-Froemel (Hrsg.), Expanding the Lexicon. Linguistic Innovation, Morphological Productivity and Ludicity, 15-42. Berlin/Boston: De Gruyter.

Filatkina, Natalia (2017): Sprachliche Konstruktion von Zukunftsangst und Misstrauen in deutschen Medien der Gegenwart. Unveröffentlichtes Manuskript zum Habilitationsvortrag an der Universität Trier.

Filatkina, Natalia (2016): Darumb kam eine seer grosse furcht vnd schrecken in das Volck. angest vs. vorhte - Martin Luther als Wendepunkt? In: Nina Bartsch \& Simone SchultzBalluft (Hrsg.), Perspektiv Wechsel oder Wiederentdeckung der Philologie. Band 2: Grenzgänge und Grenzüberschreitungen. Zusammenspiele von Sprache und Literatur in Mittelalter und Früher Neuzeit, 67-89. Berlin: Erich Schmidt.

Filatkina, Natalia (2015): Diskurshistorische Analysen des Begriffs ,Zukunftsangst‘ anhand des SPIEGEL-ONLINE-Archivs. Sprachwissenschaft 40, 79-126.

Foroutan, Naika (2016): Nationale Bedürfnisse und soziale Ängste. In: María do Mar Castro Varela \& Paul Mecheril (Hrsg.), Die Dämonisierung der Anderen. Rassismuskritik der Gegenwart, 97-107. Bielefeld: transcript.

Furedi, Frank (2007): The only thing we have to fear is the 'culture of fear' itself. http://frankfuredi.com/pdf/fearessay-20070404.pdf (12.05.2019).

Gardt, Andreas (2018): Wort und Welt. Konstruktivismus und Realismus in der Sprachtheorie. In: Ekkehard Felder \& Andreas Gardt (Hrsg.), Wirklichkeit oder Konstruktion? Sprachtheoretische und interdisziplinäre Aspekte einer brisanten Alternative, 1-44. Berlin/Boston: De Gruyter.

Gardt, Andreas (2013): Textanalyse als Basis der Diskursanalyse. Theorie und Methoden. In: Ekkehard Felder (Hrsg.), Faktizitätsherstellung in Diskursen. Die Macht des Deklarativen, 29-56. Berlin/Boston: De Gruyter. 
Georgi, Christopher (2018): Zur sprachlichen Thematisierung der Angst in Folge von Terrorismus - Eine datengeleitete Studie. In: Fabian Klinker, Joachim Scharloth \& Joanna Szczęk (Hrsg.), Sprachliche Gewalt. Formen und Effekte von Pejorisierung, verbaler Aggression und Hassrede, 109-131. Stuttgart: Metzler.

Goebel, Simon (2017): Politische Talkshows über Flucht. Wirklichkeitskonstruktionen und Diskurse. Eine kritische Analyse. Bielefeld: transcript.

Gür-Şeker, Derya (2018): Pegida as Angstneurotiker: A Linguistic Analysis of Concepts of Fear in Right-wing Populist Discourses in German Online Media. In: Elena Furlanetto \& Dietmar Meinel (Hrsg.), A Poetics of Neurosis. Narratives of Normalcy and Disorder in Cultural and Literary Texts, 115-136. Bielefeld: transcript.

Hermanns, Fritz (1995): Begriffsgeschichte als Mentalitätsgeschichte. Überlegungen zu Sinn und Form und Gegenstand historischer Semantik. In: Andreas Gardt, Klaus J. Mattheier \& Oskar Reichmann (Hrsg.), Sprachgeschichte des Neuhochdeutschen. Gegenstände, Methoden, Theorien, 69-101. Tübingen: Niemeyer.

Jung, Matthias, Martin Wengeler \& Karin Böke (Hrsg.) (1997): Die Sprache des Migrationsdiskurses. Das Reden über „Ausländer“ in Medien, Politik und Alltag. Opladen: Westdeutscher.

Kalwa, Nina (2013): Das Konzept „Islam“: Eine diskurslinguistische Untersuchung. Berlin/Boston: De Gruyter.

Koch, Lars (Hrsg.) (2013): Angst. Ein interdisziplinäres Handbuch. Stuttgart: Metzler.

Koch, Lars (2010): Das DFG-Netzwerk „Spielformen der Angst“. http://angst.werkleitz.de/fileadmin/Angst_Media/AiG/Beitraege_DEUTSCH/Vorstellung DFG_Netzwerk_Koch_web_100618gq_finalBild.pdf (12.05.2019).

Kreußler, Fabian \& Martin Wengeler (2018): Flüchtlinge, Geflüchtete oder Vertriebene? Zum Wortschatz in öffentlichen Migrationsdiskursen seit den 1950er Jahren. In: Joachim Klose \& Walter Schmitz (Hrsg.), Wer ist Deutschland? Aspekte der Migration in Kultur, Gesellschaft und Politik, 301-333. Dresden: Thelem.

Kuck, Kristin (2018a): Krisenszenarien: Metaphern in wirtschafts- und sozialpolitischen Diskursen. Berlin/Boston: De Gruyter.

Kuck, Kristin (2018b): Angst und Mut im Wahlkampf der FDP. Aptum. Zeitschrift für Sprachkritik und Sprachkultur 14/1, 36-49.

Lüger, Heinz-Helmut (2018): Zwischen Akzeptanzstützung und Pseudo-Argumentation. Phraseme im politischen Sprachgebrauch. In: Anna Gondek, Alina Jurasz \& Joanna Szczęk (Hrsg.), Einblicke und Rückblicke. Beiträge zur deutschen Phraseologie und Parömiologie aus intra- und interlingualer Sicht, 75-92. Baltmannsweiler: Schneider.

Pfeiffer, Christian (2016): Frequenz und Funktionen phraseologischer Wendungen in meinungsbetonten Pressetexten (1911-2011). Hohengehren: Schneider.

Rada, Roberta, V. (2018): Phraseme im deutschsprachigen Mediendiskurs über die Migration am Beispiel von Wir schaffen das. In: Anna Gondek, Alina Jurasz \& Joanna Szczęk (Hrsg.), Einblicke und Rückblicke. Beiträge zur deutschen Phraseologie und Parömiologie aus intra- und interlingualer Sicht, 147-161. Baltmannsweiler: Schneider.

Radeiski, Bettina (2011): Seuchen, Ängste und Diskurse. Massenkommunikation als diskursives Rollenspiel. Berlin: De Gruyter.

Römer, David (2018): Diskursphraseme- Modellierung und Beispiel. In: Sören Stumpf \& Natalia Filatkina (Hrsg.), Formelhafte Sprache in Text und Diskurs, 147-160. Berlin/Boston: De Gruyter.

Römer, David (2017): Wirtschaftskrisen. Eine Diskursgeschichte. Berlin/Boston: De Gruyter. 
Römer, David \& Martin Wengeler (2013): „Wirtschaftskrisen“ begründen / mit „Wirtschaftskrisen“ legitimieren. Ein diskurshistorischer Vergleich. In: Martin Wengeler \& Alexander Ziem (Hrsg.), Sprachliche Konstruktionen von Krisen. Interdisziplinäre Perspektiven auf ein fortwährend aktuelles Phänomen, 269-288. Bremen: Hempen.

Schmidt, Annelie (2018): „Sicherheit“ im öffentlichen Sprachgebrauch: Eine diskurslinguistische Analyse. Berlin/Boston: De Gruyter.

Seifert, Matthias \& Ulrich Brinkmann (2000): „Trau, schau, wem?“: Anmerkungen zum Mißtrauen gegenüber Fremden. In: Jennifer Wasmuth (Hrsg.), Zwischen Fremd- und Feindbildern: interdisziplinäre Beiträge zu Rassismus und Fremdenfeindlichkeit, 198-218. Münster: Lit.

Spieß, Constanze (2016): „Zäune“ oder „bauliche Maßnahmen“ für eine „Festung Europas.“ Das Sprechen über Fluchtbewegungen und Migrant*innen im öffentlich-politischen Diskurs. OBST. Osnabrücker Beiträge zur Sprachtheorie 89, 57-84.

Spieß, Constanze (2018): „Deutschland muss Deutschland bleiben“ - Sprachliche Selbst- und Fremdpositionierungsaktivitäten im Kontext politischer Äußerungen über Migration am Beispiel des Ausdrucks Leitkultur. Kulturwissenschaftliche Zeitschrift 1/2018, 35-55. https://www.degruyter.com/downloadpdf/j/kwg.2018.3.issue-1/kwg-2018-0003/kwg2018-0003.pdf (12.05.2019).

Spitzmüller, Jürgen \& Ingo Warnke (2011): Diskurslinguistik. Eine Einführung in Theorien und Methoden der transtextuellen Sprachanalyse. Berlin/Boston: De Gruyter.

Stein, Stephan \& Sören Stumpf (2019): Muster in Sprache und Kommunikation. Eine Einführung in Konzepte sprachlicher Vorgeformtheit. Berlin: Erich Schmidt.

Stumpf, Sören \& Natalia Filatkina (Hrsg.) (2018a): Formelhafte Sprache in Text und Diskurs. Berlin/Boston: De Gruyter.

Stumpf, Sören \& Natalia Filatkina (2018b): Einleitung: Formelhafte Sprache in Text und Diskurs. In: Sören Stumpf \& Natalia Filatkina (Hrsg.), Formelhafte Sprache in Text und Diskurs, 1-12. Berlin/Boston: De Gruyter.

Stumpf, Sören (2017): Ist das Zentrum-Peripherie-Modell in der heutigen Phraseologieforschung noch haltbar? Sprachwissenschaft 42 (1), 59-96.

Stumpf, Sören \& Christian D. Kreuz (2016): Phraseologie und Diskurslinguistik - Schnittstellen, Fallbeispiele und Forschungsperspektiven. Zeitschrift für Angewandte Linguistik 65, $1-37$.

Stumpf, Sören \& Christian D. Kreuz (2018): Phrasem-Bild-Beziehungen im Diskurs. Theoretische Überlegungen und methodische Ansätze zur multimodalen und diskurssemantischen Phrasem-Analyse. In: Sören Stumpf \& Natalia Filatkina (Hrsg.), Formelhafte Sprache in Text und Diskurs, 115-146. Berlin/Boston: De Gruyter.

Wengeler, Martin (2019): „Im Moment haben die Vertreter der Angstrhetorik die Lufthoheit“. Diskurslinguistische Untersuchungen zur Konstruktion von Zukunftsangst und Vertrauen in deutschen Printmedien. In: Vedad Smailagić (Hrsg.), Die Leistung der Philologie bei der Deutung der Kultur(en), 95-113. Tübingen: Stauffenburg.

Wengeler, Martin \& Alexander Ziem (Hrsg.) (2013): Sprachliche Konstruktionen von Krisen. Interdisziplinäre Perspektiven auf ein fortwährend aktuelles Phänomen. Bremen: Hempen.

Wengeler, Martin (2007): Topos und Diskurs - Möglichkeiten und Grenzen der topologischen Analyse gesellschaftlicher Debatten. In: Ingo Warnke (Hrsg.), Diskurslinguistik nach Foucault. Theorie und Gegenstände, 165-187. Berlin: De Gruyter. 
Wengeler, Martin (2006): Linguistik als Kulturwissenschaft. Eine Einführung in diesen Band. In: Martin Wengeler (Hrsg.), Linguistik als Kulturwissenschaft, 1-23. Hildesheim/New York: Olms.

Wengeler, Martin (2005): „Das Szenario des kollektiven Wissens einer Diskursgemeinschaft entwerfen“. Historische Diskurssemantik als „kritische Linguistik“. Aptum. Zeitschrift für Sprachkritik und Sprachkultur 1 (3), 262-282.

Wengeler, Martin (2003): Topos und Diskurs. Begründung einer argumentationsanalytischen Methode und ihre Anwendung auf den Migrationsdiskurs (1960-1985). Tübingen: Niemeyer.

Wirrer, Jan (1998): Phraseologismen in der Argumentation. In: Jan Wirrer (Hrsg.), Phraseologismen in Text und Kontext. Phrasemata I, 121-149. Bielefeld: Aisthesis.

Wodak, Ruth (2016): Politik mit der Angst. Zur Wirkung rechtspopulistischer Diskurse. Wien, Hamburg: Edition Konturen. 